\title{
Edge-boundary Problems with Singular Trace Conditions
}

\author{
B.-W. Schulze and Y. Wei
}

\begin{abstract}
The ellipticity of boundary value problems on a smooth manifold with boundary relies on a two-component principal symbolic structure $\left(\sigma_{\psi}, \sigma_{\partial}\right)$, consisting of interior and boundary symbols. In the case of a smooth edge on manifolds with boundary we have a third symbolic component, namely the edge symbol $\sigma_{\wedge}$, referring to extra conditions on the edge, analogously as boundary conditions. Apart from such conditions 'in integral form' there may exist singular trace conditions, investigated in [6] on 'closed' manifolds with edge. Here we concentrate on the phenomena in combination with boundary conditions and edge problem.
\end{abstract}

\section{Contents}

\section{Introduction}

1 Operators on a manifold with edge and boundary 4

1.1 Edge-degenerate families of boundary value problems . . . . . . . . 4

1.2 The regular edge calculus . . . . . . . . . . . . . . . . . . 7

2 Calculus with singular asymptotic types 12

2.1 Singular trace and Green symbols . . . . . . . . . . . . . . . 12

2.2 Global operators and ellipticity with singular trace condition . . . 16

$\begin{array}{lr}\text { References } & 20\end{array}$ 


\section{Introduction}

We study boundary value problems on a manifold $M$ with smooth edge $Y$ and boundary in the framework of a calculus of pseudo-differential operators with a principal symbolic hierarchy $\sigma=\left(\sigma_{\psi}, \sigma_{\partial}, \sigma_{\wedge}\right)$, with interior, boundary, and edge symbolic components, respectively. Such so-called edge algebras are developed in different contexts, first on 'closed' manifolds with edge, (in this case without $\sigma_{\partial}$ ), see [10], [2] and also on manifold with boundary, see [3], [8]. In such calculi the edge contributes extra trace and potential operators, which is a similar effect as in boundary value problems without the transmission property at the boundary. In contrast to the case of operators with the transmission property, see the work of Boutet de Monvel [1], the violated transmission property causes regularity of solutions to elliptic equations in weighted Sobolev spaces instead of standard ones. Thus, in general, there cannot exist local trace conditions, say, of Dirichlet or Neumann type. The trace operators are non-local, i.e., of integral form (if they exist at all for an elliptic operator). Recently there was found a way also to apply analogues of local conditions in the edge case, see the paper [6] when the manifold with edge is closed (see also [11, Section 3.2.4]).

In the present paper we investigate the case with a smooth edge on the boundary. At the same time we establish a new version of edge calculus. To illustrate the idea let us recall on how boundary conditions in the case of standard boundary value problems are organised, for simplicity, on Schwartz spaces $\mathcal{S}\left(\overline{\mathbb{R}}_{+}\right)\left(=\left.\mathcal{S}(\mathbb{R})\right|_{\overline{\mathbb{R}}_{+}}\right)$ on the inner normal $\overline{\mathbb{R}}_{+}$to the boundary. Local trace operators have the form $T: \mathcal{S}\left(\overline{\mathbb{R}}_{+}\right) \rightarrow \mathbb{C}$,

$$
T_{1} u:=\left.B u\right|_{r=0}, \quad B:=\sum_{j=0}^{N} b_{j} \partial_{r}^{j},
$$

for certain coefficients $b_{j}$ and some $N$. The pseudo-differential calculus of boundary value problems with the transmission property generates also trace operators in integral form, namely,

$$
T_{0} u=\int_{0}^{\infty} g(r) u(r) d r
$$

for some $g \in \mathcal{S}\left(\overline{\mathbb{R}}_{+}\right)$or combinations of (0.1) and (0.2) of the form $T u=$ $\int_{0}^{\infty} g(r) B u(r) d r$. Operators (0.1) require the existence of derivatives of $u$ up to the boundary such that the restriction makes sense. If such a property is not guaranteed as in the case of violated transmission property where weighted spaces (for instance $r^{-p} \mathcal{S}\left(\overline{\mathbb{R}}_{+}\right)$for some $\left.p \in \mathbb{C}\right)$ replace the ones with smoothness up to $r=0$, then we have analogues of trace operators (0.2) for suitable functions $g$ such that the integral makes sense. In the calculus itself such operators are employed as the values of operator-valued symbols along the boundary; the associated pseudodifferential operators are just the trace operators on the manifold with boundary. 
Those map functions inside to functions on the boundary. The solutions $u(r)$ to elliptic equations have asymptotics of the form

$$
u(r) \sim \sum_{j \in \mathbb{N}} \sum_{k=0}^{m_{j}} c_{j k} r^{-p_{j}} \log ^{k} r
$$

as $r \rightarrow 0$, for certain $p_{j} \in \mathbb{C}, \operatorname{Re} p_{j} \rightarrow-\infty$ as $j \rightarrow \infty$ (for simplicity at the moment we formulate things again on the half-axis). Then, instead of (0.1) we can try to pose trace conditions of the form

$$
T_{2} u=\sum_{j=0}^{N} \sum_{k=0}^{m_{j}} d_{j k} c_{j k}(u)
$$

for some coefficients $d_{j k}$ (the coefficients $c_{j k}=c_{j k}(u)$ are uniquely determined by $u$ ). It is clear that $T_{2}$ is meaningless on weighted spaces in general, but on subspaces of functions with asymptotics of some length $N$ this makes sense. This is roughly the idea of treating a part of the asymptotics as the singular one, say, for all $j$ such that $\operatorname{Re} p_{j}>\frac{1}{2}-\gamma$ for some weight $\gamma \in \mathbb{R}$, while other possible contributions to the asymptotics, i.e., for $\operatorname{Re} p<\frac{1}{2}-\gamma$ (below called regular) are embedded in spaces of weight $\gamma$ and not individually distinguished by the trace operators that only exist in this 'weight region' in integral form. It is clear that trace operator $(0.1)$ are a special case of (0.3). Analogously as in Boutet de Monvel's calculus we speak about singular trace operators. If these considerations concern a manifold with edge rather than with boundary we have to replace $\overline{\mathbb{R}}_{+}$by the non-trivial model cone $\left.X^{\Delta}=\left(\overline{\mathbb{R}}_{+} \times X\right) /\{0\} \times X\right)$ of local wedges. The base $X$ is a $C^{\infty}$ manifold, either closed compact, or with boundary. The latter is our case here, and the task is to combine the operator structures on $X$ with regular trace operators at $\partial X$ with singular trace operators with respect to the edge. This is essentially what we do in this paper. In Chapter 1 we establish the tools on operators in the calculus over $X$, organised in parameter-dependent form. Then we briefly formulate the elements of the regular edge calculus of boundary value problems in weighted spaces, with trace operators of integral form. In Chapter 2 we study singular trace operators with respect to the edge, and also singular Green operators, analogues of contributions to Green's functions in boundary value problems. Everything refers to the case of $X$ with boundary. We finally formulate the global edge algebra, and study ellipticity and parametrices within our calculus. 


\section{Operators on a manifold with edge and bound- ary}

\subsection{Edge-degenerate families of boundary value problems}

A simplest example of a manifold with edge and boundary is a wedge $M:=X^{\Delta} \times \Omega$, where $X$ is an interval on the unit circle $S^{1}, X^{\Delta}:=\left\{\tilde{x} \in \mathbb{R}^{2}: \tilde{x}=0\right.$ or $\frac{\tilde{x}}{|\tilde{x}|} \in$ $X$, for $\tilde{x} \neq 0\}$, and $\Omega \subseteq \mathbb{R}^{q}$ an open set, the edge. More generally, we can form a wedge $X^{\Delta} \times \Omega$ for any compact $C^{\infty}$ manifold $X$ with boundary; here $X^{\Delta}=\left(\overline{\mathbb{R}}_{+} \times X\right) /(\{0\} \times X)$ is the cone with base $X$, defined as a quotient space where $\{0\} \times X$ represents the tip $v$. Setting $X^{\wedge}:=\mathbb{R}_{+} \times X \cong X^{\Delta} \backslash\{v\}$ we have a splitting of variables $(r, x, y)$ in $X^{\wedge} \times \Omega$. Any other admitted splitting is associated with the given one by a diffeomorphism $\mathbb{R}_{+} \times X \times \Omega \rightarrow \mathbb{R}_{+} \times X \times \Omega$ that is the restriction of a diffeomorphism $\mathbb{R} \times X \times \Omega \rightarrow \mathbb{R} \times X \times \Omega$ to $\mathbb{R}_{+} \times X \times \Omega$ (in the sense of $C^{\infty}$ manifolds with boundary) which induces a diffeomorphism $\{0\} \times X \times \Omega \rightarrow\{0\} \times X \times \Omega$ representing at the same time an isomorphism between $X$-bundles over $\Omega$. Together with $X$ we often consider the double $2 X$ which is a smooth closed manifold, obtained by gluing together two copies $X_{ \pm}$ of $X$ along the common boundary $\partial X$ (we then identify $X_{+}$with $X$ ). Then our wedge $M:=X^{\Delta} \times \Omega$ is embedded in $2 M:=(2 X)^{\Delta} \times \Omega$ and the (closed) stretched wedge $\mathbb{M}=\overline{\mathbb{R}}_{+} \times X \times \Omega$ in $2 \mathbb{M}=\overline{\mathbb{R}}_{+} \times 2 X \times \Omega$. There is a canonical continuous map $2 \mathbb{M} \rightarrow 2 M$ induced by the projection $\overline{\mathbb{R}}_{+} \times 2 X \rightarrow(2 X)^{\Delta}$ and the identity on $\Omega$. The space $2 \mathbb{M}$ is a smooth manifold with boundary $\{0\} \times 2 X \times \Omega$ which has the structure of a (in this case trivial) $2 X$ - bundle over the edge $\Omega$. Similar constructions and observations make sense on an arbitrary manifold $M$ with smooth edge $Y$ and boundary. In this case $Y$ has a neighbourhood in $M$ which is a locally trivial $X^{\Delta}$-bundle over $Y$ for a smooth compact manifold $X$ with boundary. We then also have an associated $\overline{\mathbb{R}}_{+} \times X$-bundle over $Y$; this gives rise to the stretched manifold $\mathbb{M}$ belonging $M$. Similarly as before we have the doubles $2 \mathbb{M}$ and $2 M$, respectively, where $2 \mathbb{M}$ is a $C^{\infty}$ manifold with boundary, and $\partial(2 \mathbb{M})$ has the structure of a $2 X$-bundle over $Y$. The 'half' $\mathbb{M}$ of $2 \mathbb{M}$ has certain corners; however, since we can always pass to embedded objects in the respective double manifolds, all our constructions make sense in an invariant way also in the case with boundary.

By $\operatorname{Diff}^{\nu}(U)$ for a smooth $C^{\infty}$ manifold $U$ with boundary we denote the space of all differential operators on $U$ of order $\nu \in \mathbb{N}$ (with smooth coefficients up to the boundary). Let $\operatorname{Diff}_{\text {deg }}^{\mu}(M)$ denote the subspace of all $A \in \operatorname{Diff}^{\mu}(M \backslash Y)$ that are close to $Y$ in the variables $(r, x, y) \in X^{\wedge} \times \Omega$ of the form

$$
A=r^{-\mu} \sum_{j+|\alpha| \leq \mu} a_{j \alpha}(r, y)(-r \partial r)^{j}\left(r D_{y}\right)^{\alpha}
$$


with coefficients $a_{j \alpha} \in C^{\infty}\left(\overline{\mathbb{R}}_{+}, \operatorname{Diff}^{\mu-(j+|\alpha|)}(X)\right)$. Such operators will be called edge-degenerate.

The idea of the edge algebra from [1] of boundary value problems (BVP) is to establish an operator algebra that contains $\operatorname{Diff}_{\text {deg }}^{\mu}(M)$ together with the parametrices of elliptic elements. Since $M \backslash Y$ is a smooth manifold with boundary $\partial(M \backslash Y)$ (of course non-compact when $Y \neq \emptyset$ ) such a program extends the pseudo-differential algebra of BVP with the transmission property at the boundary.

Let us prepare our considerations by a few notions from the calculus of BVP of that kind, see also [12] or [5]. We now formulate operators on compact $X$ with boundary. By $\mathcal{B}^{\mu, d}(X)$ for $\mu \in \mathbb{Z}, d \in \mathbb{N}$, we denote the set of all operators

$$
\mathcal{A}:=\left(\mathcal{A}_{i j}\right)_{i, j=1,2}: C^{\infty}(X) \oplus C^{\infty}(\partial X) \rightarrow C^{\infty}(X) \oplus C^{\infty}(\partial X)
$$

of the form

$$
\mathcal{A}=\operatorname{diag}\left(\mathrm{r}^{+} P \mathrm{e}^{+}, 0\right)+\left(\mathcal{G}_{i j}\right)_{i, j=1,2}
$$

where $P$ is a classical pseudo-differential operator on $2 X$ of order $\mu$ with the transmission property at $\partial X$. Here $\mathrm{e}^{+}$is the operator of extension from $\operatorname{int} X_{+}$by 0 to $X_{-}$, and $\mathrm{r}^{+}$the operator of restriction to int $X_{+}$. The operator $\mathcal{G}=\left(\mathcal{G}_{i j}\right)_{i, j=1,2}$ belongs to $\mathcal{B}_{G}^{\mu, d}(X)$ and is defined as follows.

Let us first recall some notation. For any open $C^{\infty}$ manifold $\Omega$ we have $L_{(\mathrm{cl})}^{\mu}(\Omega)$, the space of (classical or non-classical) pseudo-differential operators of order $\mu \in \mathbb{R}$, based on local amplitude functions $a(x, \xi)$ of Hörmander's class $S_{1,0}^{\mu}$ and $L^{-\infty}(\Omega) \cong C^{\infty}(\Omega \times \Omega)$. We need here various operator-valued variants, referring to Hilbert spaces $H$ with group action $\varkappa=\left\{\varkappa_{\lambda}\right\}_{\lambda \in \mathbb{R}_{+}}$. By that we understand a group of isomorphisms $\varkappa_{\lambda}: H \rightarrow H$ such that $\varkappa_{\lambda} \varkappa_{\rho}=\varkappa_{\lambda \rho}$ for all $\lambda, \rho \in \mathbb{R}$, where $\lambda \rightarrow \varkappa_{\lambda} h$ defines a continuous map $\mathbb{R}_{+} \rightarrow H$ for every $h \in H$. Now if $(H, \varkappa)$ and $(\tilde{H}, \tilde{\varkappa})$ are two Hilbert spaces with group action, $U \subseteq \mathbb{R}^{p}$ open, the space

$$
S^{\mu}\left(U \times \mathbb{R}^{q} ; H, \tilde{H}\right)
$$

is defined to be the set of all $a\left(x^{\prime}, \xi^{\prime}\right) \in C^{\infty}\left(U \times \mathbb{R}^{q}, \mathcal{L}(H, \tilde{H})\right)$ such that

$$
\sup \left\langle\xi^{\prime}\right\rangle^{-\mu+|\beta|}\left\|\tilde{\varkappa}_{\left\langle\xi^{\prime}\right\rangle}^{-1}\left\{D_{x^{\prime}}^{\alpha} D_{\xi^{\prime}}^{\beta} a\left(x^{\prime}, \xi^{\prime}\right)\right\} \varkappa_{\left\langle\xi^{\prime}\right\rangle}\right\|_{\mathcal{L}(H, \tilde{H})}
$$

is finite, where sup is taken over all $x^{\prime} \in K, \xi^{\prime} \in \mathbb{R}^{q}$, for every $K \subset \subset U, \alpha \in \mathbb{N}^{p}$, $\beta \in \mathbb{N}^{q}$. A similar definition makes sense when one (or both) spaces are replaced


$E^{j}$ continuously embedded in $E^{0}$, and $\left.\varkappa\right|_{E^{j}}$ group actions in $E^{j}$ in the former sense, for all $j \in \mathbb{N}$.

A symbol $a\left(x^{\prime}, \xi^{\prime}\right)$ is called classical if there is a sequence of $a_{(\mu-j)}\left(x^{\prime}, \xi^{\prime}\right) \in$ 
$C^{\infty}\left(U \times\left(\mathbb{R}^{q} \backslash\{0\}\right) ; H, \tilde{H}\right), j \in \mathbb{N}$, with homogeneity in the sense $a_{(\mu-j)}\left(x^{\prime}, \lambda \xi^{\prime}\right)=$ $\lambda^{\mu-j} \tilde{\varkappa}_{\lambda} a_{(\mu-j)}\left(x^{\prime}, \xi^{\prime}\right) \varkappa_{\lambda}^{-1}$ for all $\lambda \in \mathbb{R}_{+}$, such that $a-\chi \sum_{j=0}^{N} a_{(\mu-j)} \in S^{\mu-(N+1)}$ for every $N \in \mathbb{N}$, with $\chi$ being any excision function in $\xi^{\prime} \in \mathbb{R}^{q}$.

Applying this to the case $H=L^{2}\left(\mathbb{R}_{+}\right), \tilde{H}:=\langle t\rangle^{-s} H^{s}\left(\mathbb{R}_{+}\right)$where $H^{s}(\mathbb{R})$ is the Sobolev space of smoothness $s \in \mathbb{R}$ on the $t$-axis $\mathbb{R}$ and $H^{s}\left(\mathbb{R}_{+}\right)=\left.H^{s}(\mathbb{R})\right|_{\mathbb{R}_{+}}$, and $\left(\varkappa_{\lambda} u\right)(t)=\lambda^{\frac{1}{2}} u(\lambda t), \lambda \in \mathbb{R}_{+}$, both in $L^{2}\left(\mathbb{R}_{+}\right)$and $\langle t\rangle^{-s} H^{s}\left(\mathbb{R}_{+}\right)$, we obtain the symbol space

$$
S_{\mathrm{cl}}^{\mu}\left(U \times \mathbb{R}^{q} ; L^{2}\left(\mathbb{R}_{+}\right), \mathcal{S}\left(\overline{\mathbb{R}}_{+}\right)\right)
$$

as the projective limit of those spaces over $s \in \mathbb{N}$. More generally, we can form

$$
S_{\mathrm{cl}}^{\mu}\left(U \times \mathbb{R}^{q} ; L^{2}\left(\mathbb{R}_{+}\right) \oplus \mathbb{C}^{k}, \mathcal{S}\left(\overline{\mathbb{R}}_{+}\right) \oplus \mathbb{C}^{l}\right)
$$

of $l \times k$-block matrices where the group actions on $\mathbb{C}^{k}$ and $\mathbb{C}^{l}$ are taken as identity for all $\lambda \in \mathbb{R}$. For simplicity set $k=l=1$ (in general $\mathbb{C}^{k}$ and $\mathbb{C}^{l}$ have the meaning of fibres of some vector bundles over the boundary). Here and in the sequel we assume $U \subseteq \mathbb{R}^{q}$ for $q=\operatorname{dim} \partial X$. Observe that the space of lower right corners in (1.5) for $k=l=1$ coincides with $S_{\mathrm{cl}}^{\mu}\left(U \times \mathbb{R}^{q}\right)$ (classical scalar symbols). An operator function $g\left(x^{\prime}, \xi^{\prime}\right) \in C^{\infty}\left(U \times \mathbb{R}^{q}, \mathcal{L}\left(L^{2}\left(\mathbb{R}_{+}\right) \oplus \mathbb{C}, \mathcal{S}\left(\overline{\mathbb{R}}_{+}\right) \oplus \mathbb{C}\right)\right.$ is called a Green symbol of order $\mu \in \mathbb{R}$ and type 0 if

$$
g\left(x^{\prime}, \xi^{\prime}\right)=\operatorname{diag}\left(1,\left\langle\xi^{\prime}\right\rangle^{\frac{1}{2}}\right) f\left(x^{\prime}, \xi^{\prime}\right) \operatorname{diag}\left(1,\left\langle\xi^{\prime}\right\rangle^{-\frac{1}{2}}\right)
$$

for some $f\left(x^{\prime}, \xi^{\prime}\right)$ in (1.5) such that also $f^{*}\left(x^{\prime}, \xi^{\prime}\right)$ (the pointwise formal adjoint) belongs to (1.5). More generally,

$$
g\left(x^{\prime}, \xi^{\prime}\right)=g_{0}\left(x^{\prime}, \xi^{\prime}\right)+\sum_{j=1}^{d} g_{j}\left(x^{\prime}, \xi^{\prime}\right) \operatorname{diag}\left(\partial_{t}^{j}, 0\right)
$$

is called a Green symbol of order $\mu$ and type $d \in \mathbb{N}$ if $g_{j}\left(x^{\prime}, \xi^{\prime}\right)$ is Green symbol of order $\mu-j$ and type 0 .

A (global) smoothing operator $\mathfrak{C}=\left(\mathfrak{C}_{i j}\right)_{i, j=1,2}$ of the class $\mathcal{B}^{-\infty, d}(X)$ is an operator of the form

$$
\mathfrak{C}=\mathfrak{C}_{0}+\sum_{k=1}^{d} \mathfrak{C}_{k} \operatorname{diag}\left(D^{k}, 0\right)
$$

where $D$ is a first order differential operator with smooth coefficients on $X$ that is close to $\partial X$ a differentiation along a vector field normal to the boundary, and the operators $\mathfrak{C}_{k}, 0 \leq k \leq d$, are $2 \times 2$ matrices of integral operators with kernels that are $C^{\infty}$ in the variables on $X \times X, X \times \partial X, \partial X \times X$, and $\partial X \times \partial X$, respectively.

Now $\mathcal{B}_{G}^{\mu, d}(X)$ is defined to be the set of all operators $\mathfrak{G}=\left(\mathfrak{G}_{i j}\right)_{i, j=1,2}$ that have the form $\mathfrak{C}$ plus a finite sum of operators $\varphi \mathfrak{G} \tilde{\varphi}$, where $\mathfrak{G}=\left(\chi^{-1}\right)_{*} \mathrm{Op}_{x^{\prime}}(g)$ for 
a chart $\chi: V \rightarrow U \times \overline{\mathbb{R}}_{+}$from a neighbourhood $V$ on $X$ intersecting the boundary, $U \subseteq \mathbb{R}^{q}$ open, $q=\operatorname{dim} \partial X$, with a Green symbol $g\left(x^{\prime}, \xi^{\prime}\right)$ of order $\mu$ and type $d$, and $\varphi, \tilde{\varphi} \in C_{0}^{\infty}(V)$. Here $\mathrm{Op}_{x^{\prime}}(g)=\mathcal{F}_{\xi^{\prime} \rightarrow x^{\prime}}^{-1} g \mathcal{F}_{x^{\prime} \rightarrow \xi^{\prime}}$ means the pseudo-differential operator with the Fourier transform $\mathrm{s}$ in $x^{\prime}$ with an amplitute function $g$ (such a notation will be used later on in other variants, as well).

We thus obtain altogether the operator space $\mathcal{B}^{\mu, d}(X)$, see the formula (1.2). It is known that every $\mathcal{A} \in \mathcal{B}^{\mu, d}(X)$ induces continuous operators

$$
\mathcal{A}: H^{s}(\operatorname{int} X) \oplus H^{s-\frac{1}{2}}(\partial X) \rightarrow H^{s-\mu}(\operatorname{int} X) \oplus H^{s-\frac{1}{2}-\mu}(\partial X) .
$$

The only change in the definition of $\mathcal{B}^{\mu, d}(X)$ for non-compact $X$ is to replace the former finite sum of operators $\varphi \mathfrak{G} \tilde{\varphi}$ by a locally finite sum. Instead of (1.8) we then obtain continuity between the respective comp/loc -variants of Sobolev spaces. There is a straightforward extension of $\mathcal{B}^{\mu, d}$ to parameter-dependent operators, i.e., to the space $\mathcal{B}^{\mu, d}\left(X ; \mathbb{R}^{l}\right)$, with $\lambda \in \mathbb{R}^{l}$ as parameter. We simply replace the former $P \in L_{\mathrm{cl}}^{\mu}(2 X)$ by $P(\lambda) \in L_{\mathrm{cl}}^{\mu}\left(2 X ; \mathbb{R}^{l}\right)$ (those operators rely on local amplitude functions $a(x, \xi, \lambda)$ with $(\xi, \lambda) \in \mathbb{R}^{n+l}$ treated as a convariable, and $\left.L^{-\infty}\left(X ; \mathbb{R}^{l}\right)=\mathcal{S}\left(\mathbb{R}^{l}, L^{-\infty}(X)\right)\right)$. Moreover, $\eta$ in (1.6) is replaced by $(\eta, \lambda) \in \mathbb{R}^{n-1+l}$ and $\mathcal{B}^{-\infty, d}(X)$ by $\mathcal{B}^{-\infty, d}\left(X ; \mathbb{R}^{l}\right):=\mathcal{S}\left(\mathbb{R}^{l}, \mathcal{B}^{-\infty, d}(X)\right)$. Here we tacitly use canonical Fréchet topologies in the respective spaces.

A family of operators $r^{-\mu} p(r, y, \rho, \eta), \mu \in \mathbb{Z}$, is called edge-degenerate if $p$ has the form $p(r, y, \rho, \eta)=\tilde{p}(r, y, r \rho, r \eta)$ for some

$$
\tilde{p}(r, y, \tilde{\rho}, \tilde{\eta}) \in C^{\infty}\left(\overline{\mathbb{R}}_{+} \times \Omega, \mathcal{B}^{-\infty, d}\left(X ; \mathbb{R}_{\tilde{\rho}, \tilde{\eta}}^{1+q}\right)\right) .
$$

\subsection{The regular edge calculus}

In order to develop the idea of singular trace and Green operators we need some details on the (regular) edge calculus of BVP on a (not necessarily compact) manifold $M$ with edge $Y$ and boundary, based on edge -degenerate families (see, for instance [5] or [3]). The structure will be similar as (1.1) and (1.2). We form operators

$$
\begin{aligned}
& C_{0}^{\infty}(M \backslash Y) \quad C^{\infty}(M \backslash Y) \\
& \oplus \quad \oplus \\
& \mathfrak{A}=\left(\mathfrak{A}_{i j}\right)_{i, j=1,2}: C_{0}^{\infty}(\partial(M \backslash Y)) \rightarrow C^{\infty}(\partial(M \backslash Y)), \\
& \oplus \\
& C_{0}^{\infty}(Y) \quad C^{\infty}(Y)
\end{aligned}
$$


$\begin{array}{ccc}C_{0}^{\infty}(M \backslash Y) & \oplus \\ \text { where } \mathfrak{A}_{11}: & \rightarrow & C^{\infty}(M \backslash Y) \\ & C_{0}^{\infty}(\partial(M \backslash Y))\end{array} \quad \begin{gathered}\oplus \\ C^{\infty}(\partial(M \backslash Y))\end{gathered} \quad$ contains elements $P \in$ $\mathcal{B}^{\mu, d}(M \backslash Y)$. From $r^{-\mu} p$ by a certain operator convention we obtain $P$ in such a way that the operator admits a continuous extension to weighted spaces. The full block matrix is given as

$$
\mathfrak{A}=\operatorname{diag}(P+M, 0)+\mathfrak{G}, \quad \mathfrak{G}=\left(\mathfrak{G}_{i j}\right)_{i, j=1,2}
$$

for a so-called smoothing Mellin operator $M$ and a matrix $\mathfrak{G}$ Green operators of the edge calculus. We define $P$ and $M$ first on a wedge neighbourhood $V$ of a point $y \in Y$, locally represented by $V=X^{\Delta} \times \Omega$ with its stretched version $\mathbb{V}=\overline{\mathbb{R}}_{+} \times X \times \Omega \ni(r, x, y)$. Let $\mathcal{M}_{O}^{\mu, d}\left(X ; \mathbb{R}^{l}\right)$ denote the space of all $h(z, \lambda) \in$ $\mathcal{A}\left(\mathbb{C}, \mathcal{B}^{\mu, d}\left(X ; \mathbb{R}^{l}\right)\right.$ ) (where $\mathcal{A}(U, E), U \subseteq \mathbb{C}$ open, is the space of all holomorphic functions in $U$ with values in a Fréchet space $E$ ) such that

$$
h(\beta+i \rho, \lambda) \in \mathcal{B}^{\mu, d}\left(X ; \mathbb{R}_{\rho, \lambda}^{1+\rho}\right)
$$

for every $\beta \in \mathbb{R}$, uniformly in compact $\beta$-intervals. Moreover, given a closed set $R \subset \mathbb{C}$ such that $R \cap\left\{c \leq \operatorname{Re} z \leq c^{\prime}\right\}$ is compact for every $c \leq c^{\prime}$, by $\mathcal{M}_{R}^{-\infty, d}(X)$ we denote the subspace of all $f \in \mathcal{A}\left(\mathbb{C} \backslash R, \mathcal{B}^{-\infty, d}(X)\right)$ such that for any $R$-excision function $\chi$ (i.e., $\chi \in C^{\infty}(\mathbb{C})$ with $\chi(z)=0$ for $\operatorname{dist}(z, R)<\varepsilon_{0}, \chi(z)=1$ for $\operatorname{dist}(z, R)>\varepsilon_{1}$ for some $\left.0<\varepsilon_{0}<\varepsilon_{1}\right)$ we have

$$
\chi f(\beta+i \rho) \in \mathcal{B}^{-\infty, d}\left(X ; \mathbb{R}_{\rho}\right)
$$

for every $\beta \in \mathbb{R}$, uniformly in compact $\beta$-intervals. The meaning of spaces $M_{R}^{-\infty, d}(X)$ for a continuous asymptotic type $R$ is analogous as that in [12, Section 2.2.4], here in the variant of BVP and $\mathcal{B}^{-\infty, d}(X)$-valued operator functions, see also [5, Section 2.1.5]. Operator functions

$$
h(r, y, z, \lambda) \in C^{\infty}\left(\overline{\mathbb{R}}_{+} \times \Omega, \mathcal{M}_{O}^{\mu, d}\left(X ; \mathbb{R}^{l}\right)\right)
$$

occur as symbols of pseudo-differential operators on $\mathbb{R}_{+}$, based on the Mellin transform $M u(z)=\int_{0}^{\infty} r^{z-1} u(r) d r$. Set $\Gamma_{\beta}:=\{z \in \mathbb{C}: \operatorname{Re} z=\beta\}$ and

$$
\mathrm{op}_{M}^{\gamma}(f)(y, \lambda) u(r)=\iint\left(\frac{r^{\prime}}{r}\right)^{-\left(\frac{1}{2}-\gamma+i \rho\right)} f\left(r, r^{\prime}, z, y, \lambda\right) u\left(r^{\prime}\right) \frac{d r^{\prime}}{r^{\prime}} d \rho
$$

for some $\gamma \in \mathbb{R}$, interpreted as a weight, $f\left(r, r^{\prime}, z, y, \lambda\right) \in C^{\infty}\left(\mathbb{R}_{+} \times \mathbb{R}_{+} \times\right.$ $\left.\Omega, \mathcal{B}^{\mu, d}\left(X ; \Gamma_{\frac{1}{2}} \times \mathbb{R}^{l}\right)\right)$, where $\rho=\operatorname{Im} z$ on $\Gamma_{\frac{1}{2}-\gamma}$ is interpreted as a component of the parameter $(\rho, \lambda)$. The operator $P$ in (1.10) is defined as an element of $\mathcal{B}^{\mu, d}(M \backslash Y)$ that is locally near $Y$ in the variables $(r, x, y) \in X^{\wedge} \times \Omega$ of the form

$$
P=\mathrm{Op}_{y}(a)
$$


where $a(y, \eta)$ is a symbol in $S^{\mu}\left(\Omega \times \mathbb{R}^{q} ; \mathcal{K}^{s, \gamma}\left(X^{\wedge}\right), \mathcal{K}^{s-\mu, \gamma-\mu}\left(X^{\wedge}\right)\right)$ between spaces $\mathcal{K}^{s, \gamma}$ to be defined below. The precise form of $a(y, \eta)$ is as follows:

$$
a(y, \eta)=\sigma r^{-\mu}\left\{\omega_{\eta} \mathrm{op}_{M}^{\gamma-\frac{n}{2}}(h)(y, \eta) \tilde{\omega}_{\eta}+\chi_{\eta} \mathrm{Op}_{r}(p)(y, \eta) \tilde{\chi}_{\eta}\right\} \tilde{\sigma}+b(y, \eta)
$$

for $p(r, y, \rho, \eta):=\tilde{p}(r, y, r \rho, r \eta)$ where $\tilde{p}(r, y, r \rho, r \eta) \in C^{\infty}\left(\overline{\mathbb{R}}_{+} \times \Omega, \mathcal{B}^{\mu, d}\left(X ; \mathbb{R}_{\tilde{\rho}, \tilde{\eta}}^{1+q}\right)\right)$, moreover, $h(r, y, z, \eta):=\tilde{h}(r, y, z, r \eta)$ where $\tilde{h}(r, y, z, \tilde{\eta}) \in C^{\infty}\left(\overline{\mathbb{R}_{+}} \times\right.$ $\left.\Omega, \mathcal{M}_{O}^{\mu, d}\left(X ; \mathbb{R}_{\tilde{\eta}}^{q}\right)\right)$ has the property

$$
\operatorname{op}_{M}^{\gamma-\frac{n}{2}}(h)(y, \eta)=\operatorname{Op}_{r}(p)(y, \eta) \quad \bmod \quad C^{\infty}\left(\Omega, \mathcal{B}^{\mu, d}\left(X^{\wedge} ; \mathbb{R}^{q}\right)\right) .
$$

(It is known that for any given $p$ such an $h$ exists, see [12]). Moreover, $\omega, \tilde{\omega}, \sigma, \tilde{\sigma}$, are cut-off functions (i.e., functions in $C_{0}^{\infty}\left(\overline{\mathbb{R}}_{+}\right)$that are equal to 1 near $r=0$ ), $\chi, \tilde{\chi}$ are excision functions, (i.e., elements of $C^{\infty}\left(\overline{\mathbb{R}}_{+}\right)$that are equal to 1 for large $r$ and vanish near zero) where $\omega+\chi=1$, and $\omega \prec \tilde{\omega}, \chi \prec \tilde{\chi}$ (e.g., $\omega \prec \tilde{\omega}$ means that $\tilde{\omega} \equiv 1$ on $\operatorname{supp} \omega)$, and $\varphi_{\eta}(r):=\varphi(r[\eta])$ for any $\varphi \in C^{\infty}\left(\mathbb{R}_{+}\right)$and any fixed function $\eta \rightarrow[\eta]$ in $C^{\infty}\left(\mathbb{R}^{q}\right)$ that is strictly positive and $|\eta|=[\eta]$ for $|\eta| \geq$ const. The operator family $b(y, \eta)$ is defined by

$$
b(y, \eta)=\varphi \mathrm{op}_{r}(B)(y, \eta) \tilde{\varphi}
$$

for some $B(r, y, \rho, \eta) \in C^{\infty}\left(\mathbb{R}_{+} \times \Omega, \mathcal{B}^{\mu, d}\left(X, \mathbb{R}^{1+q}\right)\right)$ and $\varphi, \tilde{\varphi} \in C_{0}^{\infty}\left(\mathbb{R}_{+}\right)$. The operator $M$ in (1.10) is a smoothing Mellin operator (not necessarily compact between the weighted spaces), locally near $Y$, defined as a finite linear combination of operators $\mathrm{Op}_{y}(m)$ for operator-valued symbols

$$
m(y, \eta)=r^{-\mu+j} \omega_{\eta} \mathrm{op}_{M}^{\gamma_{j \alpha}-\frac{n}{2}}\left(f_{j \alpha}\right)(y) \eta^{\alpha} \tilde{\omega}_{\eta},
$$

$j=0, \ldots, k$, with $k \in \mathbb{N}$ defining a weight interval $\Theta=(-(k+1), 0]$ (see also the role of $\Theta$ in the discussion of asymptotics below), where the ingredients are as follows: $\alpha \in \mathbb{N}^{q},|\alpha| \leq j, \gamma_{j \alpha} \in \mathbb{R}, \gamma-j \leq \gamma_{j \alpha} \leq \gamma$ for all $j, \alpha$, $f_{j \alpha}(y) \in C^{\infty}\left(\Omega, \mathcal{M}_{R_{j \alpha}}^{-\infty, d}(X)\right)$, where $R_{j \alpha} \cap \Gamma_{\frac{n+1}{2}-\gamma_{j \alpha}}=\emptyset$.

In the edge calculus of boundary value problems instead of the spaces in (1.9) we refer to weighted spaces. Those spaces are very important in the sequel. So we recall a few definitions. First we have weighted Sobolev spaces $\mathcal{H}^{s, \gamma}\left(X^{\wedge}\right)$, on the infinite cone, already employed by Kondratyev [7], in the case of a closed compact $C^{\infty}$ manifold $X$. This space for $s \in \mathbb{N}, \gamma \in \mathbb{R}$ can be defined to be the set of all $u(r, x) \in r^{\gamma-\frac{n}{2}} L^{2}\left(\mathbb{R}_{+} \times X\right)_{d r d x}, n=\operatorname{dim} X$, such that $\left(r \partial_{r}\right)^{k} D_{x}^{\alpha} u \in r^{\gamma-\frac{n}{2}} L^{2}\left(\mathbb{R}_{+} \times X\right)$ for all $k+|\alpha| \leq s$ and every $D_{x}^{\alpha} \in \operatorname{Diff}^{|\alpha|}(X)$. For $-s \in \mathbb{N}$ we obtain the definition by duality via the $\mathcal{H}^{0,0}\left(X^{\wedge}\right)$-scalar product, and for real $s$ by (complex) interpolation. Moreover, we set

$$
\mathcal{K}^{s, \gamma}\left(X^{\wedge}\right):=\omega \mathcal{H}^{s, \gamma}\left(X^{\wedge}\right)+(1-\omega) H_{\text {cone }}^{s}\left(X^{\wedge}\right),
$$


for some cut-off function $\omega(r)$, where $H_{\text {cone }}^{s}$ is defined to be the subspace of all $\left.u(r, x) \in H_{\text {loc }}^{s}(\mathbb{R} \times X)\right|_{\mathbb{R}_{+} \times X}$ such that for any coordinate neighbourhood $U$ on $X$ and a diffeomorphism $\chi: U \rightarrow U_{1}, U_{1} \subseteq S^{n}$ open, and for every $\varphi \in C_{0}^{\infty}\left(U_{1}\right)$ the function

$$
(1-\omega(r)) \varphi(x) u\left(r, \chi^{-1}(x)\right)
$$

belongs to $H^{s}\left(\mathbb{R}^{1+n}\right)$, with $(r, x) \in \mathbb{R}^{1+n} \backslash\{0\}$ being interpreted as polar coordinates in $\mathbb{R}^{1+n}$. Moreover, we set

$$
\mathcal{K}^{s, \gamma ; g}\left(X^{\wedge}\right):=\langle r\rangle^{-g} \mathcal{K}^{s, \gamma}\left(X^{\wedge}\right)
$$

for any $g \in \mathbb{R}$.

We fix a Hilbert space scalar product in $\mathcal{K}^{s, \gamma ; g}\left(X^{\wedge}\right)$ and identify $\mathcal{K}^{0,0 ; 0}\left(X^{\wedge}\right)$ with $r^{-\frac{n}{2}} L^{2}\left(\mathbb{R}_{+} \times X\right)$. The space $\mathcal{K}^{s, \gamma ; g}\left(X^{\wedge}\right)$ can be endowed with a group action $\varkappa^{g}:=\left\{\varkappa_{\lambda}^{g}\right\}_{\lambda \in \mathbb{R}_{+}}$,

$$
\varkappa_{\lambda}^{g} u(r, x):=\lambda^{\frac{n+1}{2}+g} u(\lambda r, x) .
$$

Let us set $\Theta=(\vartheta, 0],-\infty \leq \vartheta<0$, and form the Fréchet spaces

$$
\mathcal{K}_{\Theta}^{s, \gamma ; g}\left(X^{\wedge}\right):=\lim _{j \in \mathbb{N}} \mathcal{K}^{s, \gamma-\vartheta-(1+j)^{-1} ; g}\left(X^{\wedge}\right)
$$

and $\mathcal{S}_{\Theta}^{\gamma}\left(X^{\wedge}\right):=\lim _{k \in \mathbb{N}} \mathcal{K}^{k, \gamma ; k}\left(X^{\wedge}\right)$. The interval $\Theta$ measures flatness of a distribution with respect to the weight $\gamma$. It is also interesting to formulate non-trivial asymptotics rather than flatness. By that we mean, say, for finite $\vartheta$, that an element $u \in \mathcal{K}^{s, \gamma ; g}$ has a decomposition

$$
u(r, x)=u_{\text {sing }}(r, x)+u_{\text {flat }}(r, x) .
$$

Here $u_{\text {flat }} \in \mathcal{K}_{\Theta}^{s, \gamma ; g}$, and $u_{\text {sing }}$ is a singular term with, for instance, discrete asymptotics, i.e.,

$$
u_{\text {sing }}(r, x)=\omega(r) \sum_{j=0}^{N} \sum_{k=0}^{m_{j}} c_{j k}(x) r^{-p_{j}} \log ^{k} r
$$

for certain $p_{j} \in \mathbb{C}, \operatorname{Re} p_{j}<\frac{n+1}{2}-\gamma, m_{j} \in \mathbb{N}$, coefficients $c_{j k} \in C^{\infty}(X)$ and some cut-off function $\omega$. If we fix the sequence $P=\left\{\left(p_{j}, m_{j}\right)\right\}_{j=0, \ldots, N}$ we speak about a discrete asymptotic type. This gives us a Fréchet subspace $\mathcal{K}_{P}^{s, \gamma ; g}\left(X^{\wedge}\right)$ of $\mathcal{K}^{s, \gamma ; g}\left(X^{\wedge}\right)$, defined to be the set of all distributions (1.14) with $u_{\text {sing }}$ and $u_{\text {flat }}$ as just described. For purposes below we also define the space

$$
\mathcal{S}_{P}^{\gamma}\left(X^{\wedge}\right)=\lim _{j \in \mathbb{N}} \mathcal{K}_{P}^{k, \gamma ; k}\left(X^{\wedge}\right)
$$

with the Fréchet topology of the projective limit. It is clear that the group action $\varkappa^{g}$ on $\mathcal{K}^{s, \gamma ; g}\left(X^{\wedge}\right)$ induces group actions on the spaces $\mathcal{K}_{P}^{s, \gamma ; g}\left(X^{\wedge}\right)$ and $\mathcal{S}_{P}\left(X^{\wedge}\right)$. This allows us to generalise the spaces of symbols (1.5) to

$$
S_{\mathrm{cl}}^{\mu}\left(U \times \mathbb{R}^{q} ; \mathcal{K}^{s, \gamma ; g}\left(X^{\wedge}\right) \oplus \mathbb{C}^{k}, \mathcal{S}_{P}^{\gamma-\mu}\left(X^{\wedge}\right) \oplus \mathbb{C}^{l}\right),
$$


for any open set $U \subseteq \mathbb{R}^{q}$ that corresponds to a chart on our edge $Y$. In this way we will obtain an analogue of Green, trace and potential symbols explained by the formula (1.6). The order of trace and potential symbols will be interpreted here in a new way, compared with [10]. Before we do that we generalise once again the notion of discrete asymptotics by admitting so-called continuous asymptotics (similarly as before in our Mellin symbol spaces $M_{R}^{-\infty, d}(X)$ ). The main reason is that discrete asymptotic types in the edge calculus may depend on the edge variable $y$ with a very irregular behaviour of the pattern of $p_{j} \in \mathbb{C}$ and $m_{j} \in \mathbb{N}$ under varying $y$.

The idea is to describe asymptotics in terms of analytic functionals in the complex plane of the Mellin covariable. In the sequel we freely employ notation from this context, see [4], [11] or [5, Section 2.3]. Let $K \subset \mathbb{C}$ be a compact set and $C \in \mathbb{C} \backslash K$ a (say, smooth) compact curve counterclockwise surrounding $K$, with $\operatorname{dist}(C, K)<\varepsilon$ for some $\varepsilon>0$, such that the winding number of $C$ with respect to every $z \in K$ is equal to 1 (such a $C$ always exists, see [9]). If $U \subseteq \mathbb{C}$ is an open set containing an $\varepsilon$-neighbourhood of $K$, for every $f \in \mathcal{A}(U \backslash K)$ we can form a linear continuous functional $\zeta: \mathcal{A}(U) \rightarrow \mathbb{C}$ by

$$
\langle\zeta, h\rangle:=\frac{1}{2 \pi i} \int_{C} f(z) h(z) d z,
$$

$h \in \mathcal{A}(U)$. A similar construction makes sense for $f \in \mathcal{A}(U \backslash K, E)$ where $E$ is any Fréchet space. In this case we obtain $\zeta: \mathcal{A}(U) \rightarrow E$. Observe that when $K=\pi_{\mathbb{C}} P=\bigcup_{j=0}^{N}\left\{p_{j}\right\}$ and $f$ is a meromorphic $C^{\infty}(X)$-valued function with poles at the points $p_{j}$ of multiplicity $m_{j}+1$, then $\omega(r)\left\langle\zeta, r^{-z}\right\rangle$ is just of the form (1.15). This is the background of the idea of continuous asymptotics. Let $\mathcal{A}^{\prime}(K, E)$ denote the space of all $E$-valued analytic functionals, carried by the compact set $K \subset \mathbb{C}$. We say that a function $u(r, x) \in \mathcal{K}^{s, \gamma ; g}\left(X^{\wedge}\right)$ has continuous asymptotics of type $P$ (with $P$ being represented by a closed set, $P \subset\left\{\operatorname{Re} z<\frac{n+1}{2}-\gamma\right\}$, such that $P \cap\left\{c \leq \operatorname{Re} z \leq c^{\prime}\right\}$ is compact for every $\left.c \leq c^{\prime}\right)$ if for every $\beta \in(\vartheta, 0)$ there is a compact subset $K_{\beta} \subseteq P$ and an element $\zeta_{\beta} \in \mathcal{A}^{\prime}\left(K_{\beta}, C^{\infty}(X)\right)$, such that $u(r, x)=\omega(r)\left\langle\zeta_{\beta}, r^{-z}\right\rangle \bmod \mathcal{K}^{s, \gamma+\beta ; g}\left(X^{\wedge}\right)$. Clearly only $P \cap\left\{\operatorname{Re} z>\frac{n+1}{2}-\gamma+\vartheta\right\}$ affects the asymptotics which is controlled in the weight strip $\left\{\frac{n+1}{2}-\gamma+\vartheta<\operatorname{Re} z<\frac{n+1}{2}-\gamma\right\}$, since $\zeta \in \mathcal{A}^{\prime}\left(K, C^{\infty}(X)\right)$ for $K \subset\left\{\operatorname{Re} z \leq \frac{n+1}{2}-\gamma+\vartheta\right\}$ entails $\omega(r)\left\langle\zeta, r^{-z}\right\rangle \in \mathcal{K}_{\Theta}^{\infty, \gamma ; g}\left(X^{\wedge}\right)$. Similarly as before we assume that $\Theta$ is finite (the case of an infinite weight interval can be considered as well, for simplicity we do not discuss the details).

The space $\mathcal{K}_{P}^{s, \gamma ; g}\left(X^{\wedge}\right)$ of all functions with continuous asymptotics of type $P$ is a Fréchet space. Similarly as before we form the spaces $\mathcal{S}_{P}^{\gamma}\left(X^{\wedge}\right)$. Analogous notions make sense for $\partial X$ instead of $X$. We then obtain the spaces

$$
\mathcal{K}^{s, \gamma}\left(X^{\wedge}\right):=\mathcal{K}^{s, \gamma}\left(X^{\wedge}\right) \oplus \mathcal{K}^{s-\frac{1}{2}, \gamma-\frac{1}{2}}\left((\partial X)^{\wedge}\right)
$$


and subspaces

$$
\mathcal{K}_{P}^{s, \gamma}\left(X^{\wedge}\right):=\mathcal{K}_{P}^{s, \gamma}\left(X^{\wedge}\right) \oplus \mathcal{K}_{P^{\prime}}^{s-\frac{1}{2}, \gamma-\frac{1}{2}}\left((\partial X)^{\wedge}\right)
$$

of (discrete or continuous) asymptotics of type $P$. Here, for simiplicity, on the left hand side we write $P$ for both components (we could distinguish between $P$ for $X^{\wedge}$ and $P^{\prime}$ for $\left.(\partial X)^{\wedge}\right)$; however, since there are always asymptotic types with larger carriers who cover both without loss of generality we can put $\left.P=P^{\prime}\right)$. Moreover, we form $\mathcal{S}_{P}^{\gamma}\left(X^{\wedge}\right):=\mathcal{S}_{P}^{\gamma}\left(X^{\wedge}\right) \oplus \mathcal{S}_{P}^{\gamma-\frac{1}{2}}\left(X^{\wedge}\right)$. On the spaces (1.16) or the various subspaces we have the group action

$$
\varkappa_{\lambda}:=\operatorname{diag}\left(\varkappa_{\lambda}, \varkappa_{\lambda}^{\prime}\right)
$$

where $\left(\varkappa_{\lambda} u\right)(r, x)=\lambda^{\frac{n+1}{2}} u(\lambda r, x), \quad\left(\varkappa_{\lambda}^{\prime} u\right)\left(r, x^{\prime}\right)=\lambda^{\frac{n}{2}} u\left(\lambda r, x^{\prime}\right)$ for $\lambda \in \mathbb{R}_{+}$.

\section{Calculus with singular asymptotic types}

\subsection{Singular trace and Green symbols}

In the regular edge calculus of Section 1.2 we have fixed a reference weight line $\Gamma_{\frac{n+1}{2}-\gamma} \subset \mathbb{C}$ and formulated asymptotic information on weighted distributions in term of a behaviour of their Mellin transforms on the left of that line. Now we admit asymptotic types carried on the right of that weight line. Although the spaces with asymptotics themselves are as before, in our notation we distinguish between the regular part of asymptotics on the left and the so-called singular part on the right of the reference weight line; the operators of the calculus treat those parts in a different manner. Our constructions are analogous to [6]; however, here we introduce more general asymptotic types, also in the case without boundary. A singular asymptotic type will be a finite sequence

$$
S=\left\{\left(p_{j}, m_{j}\right)\right\}_{j=0, \ldots, N}
$$

with $p_{j} \in \mathbb{C}, m_{j} \in \mathbb{N}$ and $\pi_{\mathbb{C}} S=\left\{p_{0}, \ldots, p_{N}\right\} \subset\left\{\frac{n+1}{2}-\gamma<\operatorname{Re} z<\frac{n+1}{2}-(\gamma-\sigma)\right\}$ for some $\sigma>0$. Let $\omega(r)$ be a fixed cut-off function, and set

$\mathcal{E}\left(X^{\wedge}\right)_{S}:=\left\{\sum_{j=0}^{N} \sum_{k=0}^{m_{j}} \omega(r) c_{j k}(x) r^{-p_{j}} \log ^{k} r: c_{j k} \in C^{\infty}(X) \oplus C^{\infty}(\partial X) \quad\right.$ for all $\left.j, k\right\}$.

Observe that $\mathcal{E}\left(X^{\wedge}\right)_{S} \subset \mathcal{K}^{\infty, \gamma-\sigma}\left(X^{\wedge}\right)$ and $\mathcal{E}\left(X^{\wedge}\right)_{S} \cap \mathcal{K}^{s, \gamma}\left(X^{\wedge}\right)=0$ for every $s \in$ $\mathbb{R}$. The space $\mathcal{E}\left(X^{\wedge}\right)_{S}$ is Fréchet in a natural way, namely, through the isomorphism

$$
\mathcal{E}\left(X^{\wedge}\right)_{S} \cong\left(C^{\infty}(X) \oplus C^{\infty}(\partial X)\right)^{L}
$$


for $L=\sum_{j=0}^{N}\left(m_{j}+1\right)$. We now define

$$
\mathcal{K}^{s, \gamma}\left(X^{\wedge}\right)_{S}:=\mathcal{K}^{s, \gamma}\left(X^{\wedge}\right) \oplus \mathcal{E}\left(X^{\wedge}\right)_{S}
$$

in the Fréchet topology of the direct sum. (Clearly, (2.3) is independent of the choice of $\omega$.) More generally, we have $\mathcal{K}^{s, \gamma ; g}\left(X^{\wedge}\right)_{S}:=\langle r\rangle^{-g} \mathcal{K}^{s, \gamma}\left(X^{\wedge}\right)_{S}$ for any weight $g \in \mathbb{R}$ at infinity, and subspaces $\mathcal{K}_{P}^{s, \gamma ; g}\left(X^{\wedge}\right)_{S}:=\langle r\rangle^{-g} \mathcal{K}_{P}^{s, \gamma}\left(X^{\wedge}\right)_{S}$ with (continuous) asymptotics of type $P$ on the left of the weight line. For purposes below we set

$$
\mathcal{S}_{P}^{\gamma}\left(X^{\wedge}\right)_{S}:=\lim _{\leftarrow \in \mathbb{N}} \mathcal{K}_{P}^{k, \gamma ; k}\left(X^{\wedge}\right)_{S} .
$$

Let

$$
b_{S}: \mathcal{K}^{s, \gamma}\left(X^{\wedge}\right)_{S} \rightarrow \mathcal{S}\left(X^{\wedge}\right)_{S}
$$

denote the canonical projection along $\mathcal{K}^{s, \gamma}\left(X^{\wedge}\right)$. Observe that (2.4) is completely determined by the map $\mathcal{K}^{-\infty, \gamma}\left(X^{\wedge}\right) \oplus \mathcal{E}\left(X^{\wedge}\right)_{S} \rightarrow \mathcal{E}\left(X^{\wedge}\right)_{S}$. Below we employ parameter-dependent projections

$$
p_{1, S}(\eta):=\varkappa_{[\eta]} b_{S} \varkappa_{[\eta]}^{-1}, \quad p_{0, S}(\eta):=1-p_{1, S}(\eta) .
$$

Definition 2.1.1. An element

$$
t_{1}(y, \eta) \in \bigcap_{s \in \mathbb{R}} S_{\mathrm{cl}}^{\nu}\left(\Omega \times \mathbb{R}^{q} ; \mathcal{K}^{s, \gamma}\left(X^{\wedge}\right)_{S}, \mathbb{C}\right)_{\varkappa}
$$

is said to be a singular trace symbol of order $\nu$ with respect to the asymptotic type $S$ (and of type $d \in \mathbb{N}$ ) if $t_{1}(y, \eta)$ vanishes on $\mathcal{K}^{s, \gamma}\left(X^{\wedge}\right)$ for all $(y, \eta) \in \Omega \times \mathbb{R}^{q}$ and if it only depends on the coefficients

$$
c_{j k}(x):=\left(c_{j k, 1}(x), c_{j k, 2}(x)\right) \in C^{\infty}(X) \oplus C^{\infty}(\partial X)
$$

of the singular asymptotics via the action of a function $d_{j k}(y, \eta) \in C^{\infty}(\Omega \times$ $\left.\mathbb{R}^{q}, \mathcal{B}_{21,22}^{-\infty, d}(X)\right)$. Here $\left.\mathcal{B}_{21,22}^{-\infty, d}(X)\right)$ is the subspace of all $\mathcal{C} \in \mathcal{B}^{-\infty, d}(X), \mathcal{C}=$ $\left(\mathcal{C}_{i j}\right)_{i, j=1,2}$, such that $\mathcal{C}_{11}=\mathcal{C}_{12}=0$, and the action of $\left(\mathcal{C}_{21}, \mathcal{C}_{22}\right)$ on $\left(c_{j k, 1}, c_{j k, 2}\right)$ is of the form

$$
\sum_{l=0}^{d} \int_{X} d_{j k, 1}^{l}(y, \eta, x) D^{l} c_{j k, 1}(x) d x+\int_{\partial X} d_{j k, 2}\left(y, \eta, x^{\prime}\right) c_{j k, 2}\left(x^{\prime}\right) d x^{\prime}
$$

for certain $d_{j k, 1}^{l} \in C^{\infty}\left(\Omega \times \mathbb{R}^{q}, C^{\infty}(X)\right), d_{j k, 2} \in C^{\infty}\left(\Omega \times \mathbb{R}^{q}, C^{\infty}(\partial X)\right)$, and a differential operator $D$ as in (1.7).

Given a meromorphic function $v$ in the half-plane $\operatorname{Re} z>\beta$ for some real $\beta$, for a pole $p$ of multiplicity $m+1$, we set

$$
B_{p, k} v:=\frac{1}{2 \pi i} \int_{|z-p|<\varepsilon}(z-p)^{k} v(z) d z,
$$

$0 \leq k \leq m$. Here $\varepsilon>0$ is so small that $|z-p|<\varepsilon$ does not contain other poles of $v$. 
Proposition 2.1.2. Every function

$$
t(y, \eta) \in \bigcap_{s \in \mathbb{R}} C^{\infty}\left(\Omega \times \mathbb{R}^{q}, \mathcal{L}\left(\mathcal{K}^{s, \gamma}\left(X^{\wedge}\right)_{S}, \mathbb{C}\right)\right)
$$

that vanishes on $\mathcal{K}^{s, \gamma}\left(X^{\wedge}\right)$ and such that the values of $t(y, \eta)$ depend on the coefficients $c_{j k}$ involved in $\mathcal{E}\left(\boldsymbol{X}^{\wedge}\right)_{S}$ via mappings $d_{j k}(y, \eta)$ as in Definition 2.1 .1 has a representation of the form

$$
t(y, \eta)=\sum_{j=0}^{N} \sum_{k=0}^{m_{j}} d_{j k}(y, \eta) \circ B_{p_{j}, k} \circ M \omega_{1}
$$

where $\omega_{1}$ is any cut-off function and $M$ the Mellin transform, conversely (2.6) represents an element of (2.5).

Proof. If we have a function (2.5) with the assumed properties then its values are completely determined by its values on (2.2), and can be reproduced by

$$
u \rightarrow\left(B_{p_{j}, k} \circ M \omega_{1} u\right)_{0 \leq j \leq N, 0 \leq k \leq m_{j}}
$$

composed with the functions $d_{j k}(y, \eta)$. Conversely, a function $(2.6)$ belonging to (2.5) vanishes on $\mathcal{K}^{s, \gamma}\left(X^{\wedge}\right)$ and only depends on the coefficients $c_{j k}$ as described.

Remark 2.1.3. (i) The space of singular trace symbols of order $-\infty$ associated with the singular asymptotic type $S$ consist of all $t(y, \eta)$ of the form (2.5) where the coefficients $d_{j k}(y, \eta)$ depend on $\eta$ as Schwartz functions;

(ii) the homogeneous components of a singular trace symbol of order $\nu$ can be represented analogously as the right hand side of (2.5), now with coefficents $d_{j k}(y, \eta)$ that are homogeneous in $\eta \neq 0$ of corresponding orders.

This can be proved in a similar manner as Proposition 2.6. Analogously as the case without boundary, see [6], we can show that when $t(y, \eta)$ is a function on $\Omega \times\left(\mathbb{R}^{q} \backslash\{0\}\right)$ with values in $\mathcal{L}\left(\mathcal{K}^{s, \gamma}\left(X^{\wedge}\right)_{S}, \mathbb{C}\right)$, vanishing on $\mathcal{K}^{s, \gamma}\left(X^{\wedge}\right)$, then it is smooth exactly when its composition from the right with $\varkappa_{[\eta]}^{-1}$ is smooth.

By a trace symbol of order $\nu$ of the local edge calculus on the wedge $X^{\wedge} \times \Omega$ referring to the singular asymptotic type $S$ we understand an element of $S_{\mathrm{cl}}^{\nu}\left(\Omega \times \mathbb{R}^{q} ; \mathcal{K}^{s, \gamma}\left(X^{\wedge}\right)_{S}, \mathbb{C}\right)$ that has a representation

$$
t(y, \eta)=t_{0}(y, \eta) p_{0, S}(\eta)+t_{1}(y, \eta)
$$

where $t_{0}(y, \eta)$ is a trace symbol in the regular set-up (see [5, Section 4.2.3]), referring to the weight $\gamma$ and some weight interval $\Theta$, and $t_{1}(y, \eta)$ a singular trace 
symbol.

The edge calculus admits different choices of weighted analogues of Sobolev spaces. In general, if $H$ is a Hilbert space with group action $\varkappa$ (cf. the definition in Section 1.1) we have the space $\mathcal{W}^{s}\left(\mathbb{R}^{q}, H\right)$ as the completion of $\mathcal{S}\left(\mathbb{R}^{q}, H\right)$ with respect to the norm $\left\|\langle\eta\rangle^{s} \varkappa_{\langle\eta\rangle} \hat{u}(\eta)\right\|_{L^{2}\left(\mathbb{R}^{q}, H\right)}$ (if necessary we write $\mathcal{W}^{s}\left(\mathbb{R}^{q}, H\right)_{\varkappa}$, since there are different possiblities to choose $\varkappa$, and those may affect the space). Let us consider the space $\mathcal{K}^{s, \gamma ; g}\left(X^{\wedge}\right)=\langle r\rangle^{-g} \mathcal{K}^{s, \gamma}\left(X^{\wedge}\right)$ with the group action $\left(\varkappa_{\lambda}^{g} u\right)(r, x)=\lambda^{\frac{n+1}{2}+g} u(\lambda r, x), \lambda \in \mathbb{R}_{+}$. Then we obtain $\mathcal{W}^{s}\left(\mathbb{R}^{q}, \mathcal{K}^{s, \gamma ; g}\left(X^{\wedge}\right)\right)_{\varkappa^{g}}$ for every $g \in \mathbb{R}$. Analogously, we proceed with $\mathcal{K}^{s-\frac{1}{2}, \gamma-\frac{1}{2} ; g}\left((\partial X)^{\wedge}\right)=\langle r\rangle^{g} \mathcal{K}^{s-\frac{1}{2}, \gamma-\frac{1}{2}}\left((\partial X)^{\wedge}\right)$ where the group action is $v\left(r, x^{\prime}\right) \rightarrow \lambda^{\frac{n}{2}+g} v\left(\lambda r, x^{\prime}\right)=\left(\varkappa_{\lambda}^{\prime, g} v\right)\left(r, x^{\prime}\right)$. This gives us spaces

$$
\mathcal{W}^{s-\frac{1}{2}}\left(\mathbb{R}^{q}, \mathcal{K}^{s-\frac{1}{2}, \gamma-\frac{1}{2} ; g}\left((\partial X)^{\wedge}\right)\right)_{\varkappa^{\prime}, g},
$$

and we form $\mathcal{W}^{s}\left(\mathbb{R}^{q}, \mathcal{K}^{s, \gamma ; g}\left(X^{\wedge}\right)\right)_{\varkappa^{g}} \oplus \mathcal{W}^{s-\frac{1}{2}}\left(\mathbb{R}^{q}, \mathcal{K}^{s-\frac{1}{2}, \gamma-\frac{1}{2} ; g}\left((\partial X)^{\wedge}\right)\right)_{\varkappa^{\prime}, g}$ also denoted by $\mathcal{W}^{s}\left(\mathbb{R}^{q}, \mathcal{K}^{s, \gamma ; g}\left(X^{\wedge}\right)\right)$ for $s, \gamma, g \in \mathbb{R}$. Especially for $g=0$ we omit $g$ from the notation. Analogously we have the spaces

$$
\mathcal{W}^{s}\left(\mathbb{R}^{q}, \mathcal{K}_{(P)}^{s, \gamma ; g}\left(X^{\wedge}\right)_{S}\right)
$$

where subscript $(P)$ again indicates regular edge asymptotics of type $P$ (or no asymptotics on the left of the reference weight line) and $S$ singular asymptotics.

If $M$ is a (say, compact) manifold with edge $Y$ and boundary $\partial M$ we apply local decriptions by wedges $X^{\wedge} \times \mathbb{R}^{q}$ and $(\partial X)^{\wedge} \times \mathbb{R}^{q}$, respectively. Concerning the transition maps we impose a mild extra condition, namely, that those are independent of $r$ for small $r$. Using the invariance of the above-mentioned edge spaces (that are far from $r=0$ the standard Sobolev spaces) we obtain global spaces

$$
\mathcal{W}^{s, \gamma ; g}(M) \text { and } \mathcal{W}_{(P)}^{s, \gamma ; g}(M)_{S},
$$

respectively (for instance, $\mathcal{W}^{s, \gamma ; g}(M)=\mathcal{W}^{s, \gamma ; g}(M) \oplus \mathcal{W}^{s-\frac{1}{2}, \gamma-\frac{1}{2} ; g}(\partial M)$ ). In the case $g=s-\gamma$ our spaces have a particularly natural behaviour, e.g., with respect to the multiplicativity of weights (recall that the group action depends on $g$ ). For $g=s-\gamma$ we employ the notation

$$
\boldsymbol{H}^{s, \gamma}(M) \text { and } \quad \boldsymbol{H}_{(P)}^{s, \gamma}(M)_{S}
$$

rather than (2.8). For $g=0$ we omit the corresponding upper subscripts at the spaces $(2.8)$. 


\subsection{Global operators and ellipticity with singular trace con- dition}

In spaces and operators globally on a (compact) manifold $M$ with boundary $\partial M$ and edge $Y$ it is useful to associate global operators $P_{0, S}$ with the symbols $p_{0, S}(\eta)$ defined in Section 2.1.

First note that the spaces (2.7) (for $g=0$, and without subscript $(P)$ ) can be written in the form

$$
\mathcal{W}^{s}\left(\mathbb{R}^{q}, \mathcal{K}^{s, \gamma}\left(X^{\wedge}\right)_{S}\right)=\mathcal{W}^{s}\left(\mathbb{R}^{q}, \mathcal{K}^{s, \gamma}\left(X^{\wedge}\right)\right) \oplus \mathcal{V}^{s}\left(\mathbb{R}^{q}, \mathcal{E}_{S}\left(X^{\wedge}\right)\right)
$$

for

$$
\mathcal{V}^{s}\left(\mathbb{R}^{q}, \mathcal{E}_{S}\left(X^{\wedge}\right)\right)=\mathcal{F} \varkappa_{\langle\eta\rangle} \mathcal{F} H^{s}\left(\mathbb{R}^{q}, \mathcal{E}_{S}\left(X^{\wedge}\right)\right) \oplus \mathcal{F} \varkappa_{\langle\eta\rangle}^{\prime} \mathcal{F} H^{s-\frac{1}{2}}\left(\mathbb{R}^{q}, \mathcal{E}_{S}\left((\partial X)^{\wedge}\right)\right)
$$

Then $\operatorname{Op}\left(p_{0, S}\right)$ is the canonical projection to $\mathcal{W}^{s}\left(\mathbb{R}^{q}, \mathcal{K}^{s, \gamma}\left(X^{\wedge}\right)\right)$. Analogously as in the case without boundary (see [6, Theorem 6.2]) we form an operator $P_{0, S} \in \mathcal{L}\left(\mathcal{W}^{s, \gamma}(M)_{S}\right)$ which is a projection to $\mathcal{W}^{s, \gamma}(M)$, applying the local expressions $\mathrm{Op}\left(p_{0, S}\right)$, combined with pull backs under charts to local wedges and some partition of untiy on $M$. There are different choices of such operators, but the difference is and a singular Green operator of order -1 that maps to functions of infinite flatness at the edge (first locally and then in the sense of our global definition below). A smoothing operator (of type $d \in \mathbb{N}$ along the smooth part of $\partial M)$ associated with asymptotic types $S$ and $T$ and with weight data $(\gamma, \gamma-\mu)$ is an operator



which is continuous for all $s \in \mathbb{R}, s>d-\frac{1}{2}$, where $P_{0, S}$ is a projection of the above mentioned kind, and $\mathcal{C}_{0}$ is a of an analogous structure as smoothing operator from the regular edge calculus of type $d$ with continuous asymptotics close to $Y$ (see [5], here mapping to edge spaces with singular asymptotics of type $T$ and regular asymptotics of type $P$, see also [6]) while $C_{11}$ and $C_{21}$ vanish on $\mathcal{W}^{s, \gamma}(M)$, and there is a continuous asymptotic type $P$ such that $C_{11}=\sum_{j=0}^{d} C_{11, j} \operatorname{diag}\left(D^{j}, 0\right)$ where

$$
C_{11, j}: \mathcal{W}^{s, \gamma}(M) \rightarrow \mathcal{W}_{P}^{\infty, \gamma-\mu}(M)_{T}
$$

for an operator $D$ analogously as in (1.7), and $C_{21}=\sum_{j=0}^{d} C_{21, j} \operatorname{diag}\left(D^{j}, 0\right), C_{21, j}$ : $\mathcal{W}^{s, \gamma}(M) \rightarrow H^{\infty}(Y)$, for $s>d-\frac{1}{2}$. A Green operator on $M$ of order $\nu \in \mathbb{R}$ and type $d \in \mathbb{N}$, associated with singular asymptotic types $S$ and $T$, is an operator of the form $\mathcal{G}=\mathcal{G}_{1}+\mathcal{C}$ where $\mathcal{C}$ is smoothing as just described while $\mathcal{G}_{1}$ is a finite sum of $3 \times 3$ block matrix operators defined by $\mathrm{Op}(\mathrm{g})$ together with localising factors by a partition of unity and pulled back to the manifold, where $g \in \mathcal{R}_{G}^{\nu, d}\left(\Omega \times \mathbb{R}^{q},(\gamma, \gamma-\right.$ $\mu))_{S, T}, \Omega \subseteq \mathbb{R}^{q}$. 
Definition 2.2.1. By $\mathcal{Y}^{\mu, d}(M,(\gamma, \gamma-\mu))_{S, T}$ we denote the space of all operators of the form

$$
\mathcal{A}=\left(\begin{array}{cc}
A P_{0, S} & 0 \\
0 & 0
\end{array}\right)+\mathcal{G}
$$

where $\mathcal{G}$ is a Green operator of order $\mu$ and type $d$, associated with the singular asymptotic types $S, T$, and $A$ is an operator in the regular edge calculus on $M$ of order $\mu$ and type $d$, both associated with the weight data $(\gamma, \gamma-\mu)$ (and continuous asymptotics on the left of the reference weight lines).

Applying the general continuity properties of pseudo-differential operators with operato-valued symbols we obtain that every $\mathcal{A} \in \mathcal{Y}^{\mu, d}(M,(\gamma, \gamma-\mu))_{S, T}$ induces continuous operators

$$
\begin{array}{ccc}
\mathcal{W}^{s, \gamma}(M)_{S} & & \mathcal{W}^{s-\mu, \gamma-\mu}(M)_{T} \\
\oplus & \rightarrow & \oplus \\
H^{s}(Y) & & H^{s-\mu}(Y)
\end{array}
$$

for $s>d-\frac{1}{2}$. Operators $\mathcal{A} \in \mathcal{Y}^{\mu, d}(M,(\gamma, \gamma-\mu))_{S, T}$ have a principal symbolic structure with three components, namely,

$$
\sigma(\mathcal{A})=\left(\sigma_{\psi}(\mathcal{A}), \sigma_{\partial}(\mathcal{A}), \sigma_{\wedge}(A)\right)
$$

where $\sigma_{\psi}(\mathcal{A}) \in C^{\infty}\left(T^{*}(M \backslash Y) \backslash 0\right)$ is the standard homogeneous principal symbol of the upper left corner belonging to $L_{\mathrm{cl}}^{\mu}(\operatorname{int}(M \backslash Y))$, moreover, $\sigma_{\partial}(\mathcal{A})$ is the homogeneous principal boundary symbol of the $(2 \times 2)$ upper left corner of $\mathcal{A}$ belonging to $\mathcal{B}^{\mu, d}(M \backslash Y)$, and $\sigma_{\wedge}(\mathcal{A})$ is the homogeneous principal edge symbol, namely

$$
\sigma_{\wedge}(\mathcal{A})=\operatorname{diag}\left(\sigma_{\wedge}(A) \sigma_{\wedge}\left(P_{0, S}\right), 0\right)+\sigma_{\wedge}(\mathcal{G})
$$

where $\sigma_{\wedge}(A)$ is the edge symbol of $A$ in the sense of the regular edge calculus of BVP, moreover, $\sigma_{\wedge}\left(P_{0, S}\right)$ is locally defined by the above-mentioned operator functions $p_{0, S}$, and $\sigma_{\wedge}(\mathcal{G})$ comes from the principal homogeneous components of the classical symbols in $(y, \eta)$ involved in $\mathcal{G}$. Definition 2.2.1 has a straightforward extension to the case of operators between distributional sections of vector bundles on $M, \partial M$, and $Y$, respectively. Assuming, for simplicity, the bundles over $M$ to be trivial and of fibre dimension 1, we have corresponding pairs of bundles $\left(G_{-}, G_{+}\right)$ over $\partial M \backslash Y$ and $\left(J_{-}, J_{+}\right)$over $Y$. In this case the boundary symbol is a family of operators

$$
\sigma_{\partial}(\mathcal{A}): H^{s}\left(\mathbb{R}_{+}\right) \oplus G_{-, m^{\prime}} \rightarrow H^{s-\mu}\left(\mathbb{R}_{+}\right) \oplus G_{+, m^{\prime}},
$$

$s>d-\frac{1}{2}$, parametrised by the point of $T^{*}(\partial M \backslash Y) \backslash 0$, where $G_{ \pm, m^{\prime}}$ is the fibre of $G_{ \pm}$over a point $m^{\prime} \in \partial M \backslash Y$. Moreover, the edge symbol represents a family of continuous operators

$$
\sigma_{\wedge}(\mathcal{A})(y, \eta): \mathcal{K}^{s, \gamma}\left(X^{\wedge}\right)_{S} \oplus J_{-, y} \rightarrow \mathcal{K}^{s-\mu, \gamma-\mu}\left(X^{\wedge}\right)_{T} \oplus J_{+, y}
$$


$s>d-\frac{1}{2}$, parametrised by points in $T^{*} Y \backslash 0$, with $J_{ \pm, y}$ being the fibre of $J_{ \pm}$over $y \in Y$. The notation $\mathcal{K}^{s, \gamma}\left(X^{\wedge}\right)_{S}$ is now to be interpreted in the sense of spaces with the respective bundles in the second components.

Theorem 2.2.2. Let $\mathcal{A} \in \mathcal{Y}^{\mu, d}(M,(\gamma-\nu, \gamma-\mu-\nu))_{R, T}, \mathcal{B} \in \mathcal{Y}^{\nu, e}(M,(\gamma, \gamma-\nu))_{S, R}$ be operators in our edge operator spaces (where the involved bundles in the middle fit together). Then we have

$$
\begin{gathered}
\qquad \mathcal{A B} \in \mathcal{Y}^{\mu+\nu, h}(M,(\gamma, \gamma-(\mu+\nu)))_{S, T} \\
\text { for } h=\max \left((d+\nu)^{+}, c\right), \text { with } \rho^{+}=\max (\rho, 0) \text {, and } \\
\quad \sigma_{\psi}(\mathcal{A B})=\sigma_{\psi}(A) \sigma_{\psi}(B), \quad \sigma_{\partial}(\mathcal{A B})=\sigma_{\partial}(A) \sigma_{\partial}(B), \quad \sigma_{\wedge}(\mathcal{A B})=\sigma_{\wedge}(A) \sigma_{\wedge}(B) .
\end{gathered}
$$

Proof. The details are voluminous but of a relatively simple structure. The proof essentially consists of a combination of arguments from the standard pseudodifferential calculus over the open manifold $(M \backslash Y)$ int which gives us the symbolic rule for $\sigma_{\psi}$, the calculus of pseudo-differential boundary value problems over the smooth manifol $M \backslash Y$ with boundary with the symbolic rule for $\sigma_{\partial}$, and the edge calculus in the variant of local model cones with boundary which gives us the symbolic rule for $\sigma_{\wedge}$. Those elements are well known, see, for instance, [5] or [3] for $\sigma_{\partial}$ and $\sigma_{\wedge}$. The new aspects concern a neighbourhood of the edge $Y$ where the operators are locally described by standard pseudo-differential operators in $\mathbb{R}^{q}$, here with $3 \times 3$ block matrix valued amplitude functions taking values in the cone calculus of BVP over $X^{\wedge}$ with singular trace and Green operators. These amplitude functions are operator-valued symbols of a similar structure as (1.3), with $H, \tilde{H}$ being replaced by the spaces in (2.9). The symbolic estimates are of the kind $(1.4)$, here with $(y, \eta)$ instead of $\left(x^{\prime}, \xi^{\prime}\right)$. Such pseudo- differential operators can be composed within the corresponding calculus as is known in abstract terms from [11] (see also [3, Section 1.2]). The specific novelty in our context is that the compositions preserve the individual structure of the involved symbols, namely, to be families of pseudo-differential boundary problems on $X^{\wedge}$ with singular trace etc. entries. The latter ingredients are hidden in Green operators (apart from smoothing operators) and given in terms of classical symbols. Their pointwise composition behaviour can be characterised in a similar manner as that of operators in the cone algebra on $X^{\wedge}$, modified by extra entries in the block matrices coming from the involved singular asymptotic types, see, analogously, Definition 6.2.10 and Remark 6.2.11 in [3].

We now impose an additional condition on the singular asymptotic types $S$, namely, to be represented by a sequence of triples $S=\left\{\left(p_{j}, m_{j}, L_{j}\right)\right\}_{j=0, \ldots, N}$ with $\left\{\left(p_{j}, m_{j}\right)\right\}_{j=0, \ldots, N}$ as before but spaces $L_{j}:=L_{j}(X) \oplus L_{j}^{\prime}(\partial X)$ for finite-dimensional subspaces $L_{j}(X) \subset C^{\infty}(X), L_{j}^{\prime}(\partial X) \subset C^{\infty}(\partial X)$. We form $\mathcal{E}\left(X^{\wedge}\right)_{S}$ similarly as $(2.1)$, now for $c_{j k} \in L_{j}, 0 \leq k \leq m_{j}$, rather than 
$c_{j k} \in C^{\infty}(X) \oplus C^{\infty}(\partial X)$. In this way the space $\mathcal{E}(X)_{S}$ is finite dimensioin. Concerning other singular asymptotic types $T, \ldots$, we make an analogous assumption. All notions and constructions from Section 2.1 on have a straightforward modification; we employ them in the rest of this paper, without changing notation.

Definition 2.2.3. An operator $\mathcal{A} \in \mathcal{Y}^{\mu, d}(M,(\gamma, \gamma-\mu))_{S, T}$ is called elliptic if $\sigma_{\psi}(\mathcal{A})$ does not vanish on $T^{*}\left((M \backslash Y)_{\mathrm{int}}\right) \backslash 0$, and, in the splitting of variables $(r, x, y)$ near $Y$,

$$
\tilde{\sigma}_{\psi}(\mathcal{A})(r, x, y, \rho, \xi, \eta)=r^{\mu} \sigma_{\psi}(\mathcal{A})\left(r, x, y, r^{-1} \rho, \xi, r^{-1} \eta\right) \neq 0
$$

for $(\rho, \xi, \eta) \neq 0$, up to $r=0$, moreover, $\sigma_{\partial}(\mathcal{A})$, is bijective as an operator function (2.9) parametrised by the points of $T^{*}(\partial(M \backslash Y)) \backslash 0$, and, in the splitting of variables $\left(r, x^{\prime}, y\right)$ near $Y$

$$
\tilde{\sigma_{\partial}}(\mathcal{A})\left(r, x^{\prime}, y, \rho, \xi^{\prime}, \eta\right)=r^{\mu} \sigma_{\partial}(\mathcal{A})\left(r, x^{\prime}, y, r^{-1} \rho, \xi^{\prime}, r^{-1} \eta\right)
$$

is bijective for $\left(\rho, \xi^{\prime}, \eta\right) \neq 0$, up to $r=0$, and finally $\sigma_{\wedge}(\mathcal{A})$ is bijective as an operator function $(2.10)$, parametrised by $(y, \eta) \in T^{*} Y \backslash 0$.

To have a convenient notation, if the first (first plus second) condition of Definition holds we call the operator $\mathcal{A} \sigma_{\psi}$-elliptic $\left(\left(\sigma_{\psi}, \sigma_{\partial}\right)\right.$-elliptic).

Theorem 2.2.4. An elliptic operator $\mathcal{A} \in \mathcal{Y}^{\mu, d}(M,(\gamma, \gamma-\mu))_{S, T}$ has a parametrix $\mathcal{P} \in \mathcal{Y}^{-\mu,(d-\mu)^{+}}(M,(\gamma-\mu, \gamma))_{T, S}$, i.e., $\mathcal{P} \mathcal{A}-\mathcal{I}$ and $\mathcal{A} \mathcal{P}-\mathcal{I}$ are smoothing in the operator classes with singular asymptotic types $S, S^{\prime}$ and $T, T^{\prime}$, respectively. Moreover, $\mathcal{A}$ induces a Fredholm operator

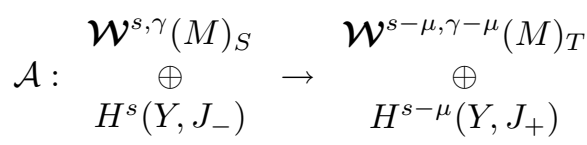

for every $s>\max (d, \mu)-\frac{1}{2}$ (similarly as in (2.10) the bundles $G_{-}$and $G_{+}$are included in the meaning of the spaces $\mathcal{W}^{s, \gamma}$ and $\mathcal{W}^{s-\mu, \gamma-\mu}$, respectively).

Proof. Writing $\mathcal{A}$ as in Definition 2.2.3 the operator $A$ in the upper left corner is elliptic in $\mathcal{B}^{\mu, d}(M \backslash Y)$ on the (non-compact) $C^{\infty}$ manifold $M \backslash Y$ with smooth boundary $\partial(M \backslash Y)$. As such it has a parametrix in $\mathcal{B}^{-\mu,(d-\mu)^{+}}(M \backslash Y)$. This will be an ingredient of the parametrix of $\mathcal{A}$ itself, namely, the part near the edge $Y$, multiplied by a function that vanishes near $Y$. It remains to construct $\mathcal{P}$ close to the edge and to add the result to the former one after a multiplication by a cut-off function that is equal to 1 near $Y$.

Let us now make some concluding remarks. As noted in the introduction certain typical effects can be read off from the case of operators on the halfaxis which is a part of the edge (or boundary) symbolic structure anyway. The 
possibility of posing boundary conditions of the form (0.1) in elliptic BVP with transmission property is a result of knowing that solutions are of sufficiently large Sobolev smoothness when we assume a corresponding smoothness of the right hand sides. However, in BVP on a manifold with singularities we may have singular solutions, as we easily see in the case of an equation $A u=f$ for $A=r^{-\mu} \sum_{j=0}^{\mu} a_{j}\left(-r \partial_{r}\right)^{j}$, say, with constants $a_{j}$. Writing $A=r^{-\mu} \mathrm{op}_{M}(h)$ for $h(z)=\sum a_{j} z^{j}$, under the assumption $h(z) \neq 0$ on $\Gamma_{\frac{1}{2}}$ we obtain solutions in the form of $u(r)=\operatorname{op}_{M}\left(h^{-1}\right)\left(r^{\mu} f\right)$. If we take the space $L^{2}\left(\mathbb{R}_{+}\right)$with the weight $\gamma=0$ as the reference space then $u(r)$ acquires asymptotics from the poles of the meromorphic function $h^{-1} M\left(r^{\mu} f\right)$ on the left of the weight line $\Gamma_{\frac{1}{2}}$. However, if there are points $p \in \mathbb{C}, \operatorname{Re} p>\frac{1}{2}$, with $h(p)=0$, then there are solutions not belonging to $L^{2}\left(\mathbb{R}_{+}\right)$. Those $p$ on the right of the reference weight line generate functions with singular asymptotics. Those cause singular trace operators, apart form regular ones referring to the left of the weight line. We see that the nature of singular asymptotics depend on the individual operator. Thus, in order to pose singular trace conditions in a concrete case we need to complete the non-bijectivity points of conormal symbols explicity. This is a separate task for every concrete operator and subject for separate papers. In [3] there are explicitly characterised those points for a large variety of BVP, in fact mixed problems with the edge as the interface where the boundary conditions have their jump.

\section{References}

[1] L. Boutet de Monvel, Boundary problems for pseudo-differential operators, Acta Math. 126 (1971), 11-51.

[2] S. Coriasco and B.-W. Schulze, Edge problems on configurations with model cones of different dimensions, Osaka J. Math. 43 (2006), 1-40.

[3] G. Harutjunjan and B.-W. Schulze, Elliptic mixed, transmission and singular crack problems, European Mathematical Soc., Zürich, 2007.

[4] L. Hörmander, The analysis of linear partial differential operators, vol. 1 and 2, Springer-Verlag, New York, 1983.

[5] D. Kapanadze and B.-W. Schulze, Crack theory and edge singularities, Kluwer Academic Publ., Dordrecht, 2003.

[6] D. Kapanadze, B.-W. Schulze, and J. Seiler, Operators with singular trace conditions on a manifold with edges, Integral Equations and Operator Theory 61 (2008), 241-279.

[7] V.A. Kondratyev, Boundary value problems for elliptic equations in domains with conical points, Trudy Mosk. Mat. Obshch. 16 (1967), 209-292. 
[8] X. Liu and B.-W. Schulze, Ellipticity on manifolds with edges and boundary, Monatshefte für Mathematik 146, 4 (2005), 295-331.

[9] W. Rudin, Real and complex analysis, McGraw-Hill, New York, 1966.

[10] B.-W. Schulze, Pseudo-differential operators on manifolds with edges, Symp. "Partial Differential Equations", Holzhau 1988, Teubner-Texte zur Mathematik, vol. 112, Teubner, Leipzig, 1989, pp. 259-287.

[11] B.-W. Schulze, Pseudo-differential operators on manifolds with singularities, North-Holland, Amsterdam, 1991.

[12] B.-W. Schulze, Boundary value problems and singular pseudo-differential operators, J. Wiley, Chichester, 1998. 